\title{
OSIRIS-THE OPTICAL, SPECTROSCOPIC AND INFRARED REMOTE IMAGING SYSTEM FOR THE ROSETTA ORBITER
}

N. Thomas*, H. U. Keller*, E. Arijs**, C. Barbieri***, M. Grande ${ }^{\frac{1}{4}}$, P. Lamy ${ }^{\frac{1}{5}}$, H. Rickman ${ }^{6}$, R. Rodrigo ${ }^{7}$, K.-P. Wenzel ${ }^{8}$, M. F. A'Hearn ${ }^{9}$, F. Angrilli***, M. Bailey ${ }^{10}$, M. A. Barucci ${ }^{11}$, J.-L. Bertaux ${ }^{12}$, K. Brieß ${ }^{13}$, J. A. Burns ${ }^{14}$, G. Cremonese***, W. Curdt*, H. Deceuninck**, R. Emery ${ }^{\dagger}$, M. Festou ${ }^{15}$, M. Fulle ${ }^{16}$, W.-H. Ip*, L. Jorda*, A. Korth*, D. Koschny*, J.-R. Kramm*, E. Kührt ${ }^{13}$, L. M. Lara ${ }^{17}$, A. Llebaria ${ }^{+}$, J. J. Lopez-Moreno ${ }^{17}$, F. Marzari***, D. Moreau**, C. Muller**, C. Murray ${ }^{18}$, G. Naletto***, D. Nevejans**, R. Ragazzoni***, L. Sabau ${ }^{19}$, A. Sanz ${ }^{20}$, J.-P. Sivan ${ }^{21}$ and G. Tondello***

\begin{abstract}
The scientific objectives, design, and implementation of the Optical, Spectroscopic, and Infrared Remote Imaging System (OSIRIS) for the International Rosetta Mission are described. The instrument comprises two camera systems with a common electronics box. A narrow angle camera will provide high resolution images of the structure and morphology of the nucleus of a comet. A wide angle camera with high straylight rejection and dynamic range will be used to investigate the innermost coma and the emission process at the surface of the comet. An infrared imaging system, which dramatically enhances the scientific return has been included in the narrow angle camera at little extra cost.
\end{abstract}


The International Rosetta Mission (hereafter "Rosetta") of the European Space Agency (ESA) is due to be launched to the short-period comet, 46P/Wirtanen, in January 2003 . A payload comprising about $120 \mathrm{~kg}$ of experiments has been pre-selected to investigate the nucleus of the target comet as the spacecraft approaches to within a few nuclear radii of the surface. In addition, it is currently envisaged that the payload will be augmented by the inclusion of two surface science packages (SSPs), RoLand and Champollion, which will be dropped onto the nucleus during a close approach to the surface.

In response to the Announcement of Opportunity (AO) (ESA document, 1996), the Optical, Spectroscopic, and Infrared Remote Imaging System (OSIRIS) was the only experiment proposed to ESA as the main imaging system on the Rosetta spacecraft. The "Red Report" (ESA document, 1993), which outlined the goals and implementation of the Rosetta mission, included a dedicated scientific imaging system as part of the strawman payload. However, funding problems have led to considerable uncertainty as to whether the ESA Member States can fund such a system completely. These problems have not, at time of writing (July 1996) been resolved. However, continuing studies have been approved through to March 1997 and indications are that a version of OSIRIS, slightly descoped from the original proposal, will take its place in the Rosetta payload.

In this paper, the current baseline for the OSIRIS experiment is described. The following sections briefly present the fundamental concept behind the experiment, the scientific objectives, the designs of the two camera systems, and the IR option. The paper concludes with a summary of the designed instrument performance.

\section{INSTRUMENT CONCEPT}

The Halley Multicolour Camera (HMC) on ESA's Giotto spacecraft provided the most detailed imaging observations of the nucleus during a fast $\left(68.37 \mathrm{~km} \mathrm{~s}^{-1}\right)$ fly-by of comet P/Halley on March 14, 1986 (Keller et al., 1996). The images revealed a large, ellipsoidal, nucleus $\left(15.3 \times 7.4 \times 7.2 \mathrm{~km}^{3}\right)$ with a low geometric albedo $\left(0.05 \pm_{0.01}^{0.03}\right)$ and slightly red $\left(\approx 6 \%(100 \mathrm{~nm})^{-1}\right)$ colour. Dust emission was evident only from discrete areas comprising $10-15 \%$ of the surface. Surface features could be seen and structures in the dust emission were apparent. The images answered fundamental questions about the nature of cometary nuclei and the source of dust and gas by showing the existence of a single solid nucleus as originally envisaged by Whipple (1950). However, the comparatively low resolution of the images $\left(>50 \mathrm{~m} \mathrm{px}^{-1}\right)$ and the limited time within the inner coma ( $\approx 15$ minutes) meant that detailed questions on the structure of cometary nuclei and the nature of their activity remained unanswered. In attempting to answer these questions, the objectives of an imaging system in close proximity to a cometary nucleus fall into two broad categories.

On the one hand, a high resolution imaging system is needed to investigate the nucleus itself. This system would attempt to find characteristic scale lengths which would indicate how the nucleus formed. It would look at how the physical properties of the surface lead to activity and how this activity affects the structure of the surface.

On the other hand, a wide field of view (FOV) system is needed to investigate the physical properties of the outflow of dust and gas from the nucleus. Although the fundamental equations describing the dynamics of dusty gas jets are fairly well established (Knollenberg, 1994, Crifo et al. 1996), there are several aspects of the comet P/Halley observations which remain unresolved. This may well result from our limited knowledge of the nature of the source and the physics of the boundary layer.

To address these two, very different, objectives, the OSIRIS experiment consortium have proposed a dual camera system along the lines suggested in the Red Report. A narrow-angle camera (NAC) with high 
resolution has been designed and optimised to study the nucleus. A wide-angle camera (WAC) has been designed to view large parts of the inner coma and investigate dust and gas emission directly above the surface of the nucleus. Once this natural division has been made the scientific objectives for each system suggest themselves.

\section{SCIENTIFIC OBJECTIVES}

The primary scientific goals of the NAC are to

- determine the rotational state of the nucleus and its moments of inertia

- determine the volume and bulk density of the nucleus

- $\quad$ search for residual evidence of formation mechanisms and scale lengths

- $\quad$ investigate topographical features and associated physical processes

- map surface variegation (ices, organics, inert surfaces)

- investigate the colour and mineralogy of the surface regolith and the degree of inhomogeneity

- determine the mass loss rate and estimate the magnitude of non-gravitational forces

- characterise the landing site(s) of the SSP(s)

- analyse short term variability, outbursts, and crustal diffusion processes

- search for gravitationally-bound material (including large particles which might endanger the spacecraft).

The WAC will be used to

- identify and quantify all sources of dust emission and measure their variation with time

- $\quad$ search for evidence of particle fragmentation, acceleration, condensation, and optical depth effects close to the dust source

- determine the near-surface flow-field of dust and its temporal evolution

- determine the optical and physical properties of the dust

- quantify night-side activity and thermal inertia effects on emission

- estimate the dust particle size distribution

- compare the distribution of daughter molecules with parents and evaluate the usefulness of daughter molecules as tracers for parent species

- investigate the chemical homogeneity of active regions.

During the conceptual design phase, it was recognised that several important scientific objectives could not be achieved with the strawman payload proposed in the Red Report. These objectives were to

- map specific ices on the surface

- map the flow-field of parent molecules in the immediate vicinity of active regions

- determine the spatial distribution of the sources of parent molecules within active regions

- determine the magnitude and distribution of extended sources of parent and daughter molecules and their influence on the inner coma flow field

- identify minerals on the surface

- mapping of the $217.5 \mathrm{~nm}$ interstellar absorption feature (if present)

- compare the collisionally excited IR emission of CO with the fluorescent UV emission of $\mathrm{CO}$ and resolve the collisional mean free path.

It was also recognised that by incorporating some modifications to the NAC and WAC designs, many of these objectives could be achieved. Thus, in the initial proposal, several options were presented which would increase the scientific return at the cost of increased complexity but only small increases in mass and power. Of these options, the proposed UV spectrographs have been superceded by the inclusion of a dedicated UV spectrometer (ALICE) in the Rosetta payload. The proposed IR imaging system (described below) remains at this stage subject to a demonstration that the relevant scientific objectives can be achieved within the 
spacecraft resources using passive cooling.

\section{NAC AND WAC REQUIREMENTS}

\section{Straylight and over-exposure control.}

It is necessary to use the scientific objectives to derive instrument parameters. In view of the wide range of targets and illumination levels in the mission, both the NAC and WAC are required to be framing chargecoupled device (CCD) cameras with mechanical shutters. The optical design has paid close attention to the question of dynamic range within an image. A key investigation will be the study of gas and dust directly above the limb. Allowing the nucleus to remain on the CCD while exposing for the dust and gas emission above the limb is highly desirable but demands high straylight rejection and over-exposure control. To achieve this, off-axis telescope designs have been selected (eliminating the scattering from the central obscuration found in Ritchey-Chretien or Cassegrain designs). Anti-blooming CCDs have been rejected because of the risks of new development. Instead, a new technique known as "clock dithering", which manipulates the clock levels on the detector to promote recombination of blooming charge, will be used to allow over-exposure on parts of the CCD while achieving good signal to noise data on low surface brightness regions in the frame.

Image scale of the NAC.

The image scale for the systems has required careful consideration. The resolution of the NAC is driven by several scientific objectives and operational constraints. Inhomogeneity over small scale lengths could potentially bias the results from the SSPs. Therefore, it is mandatory to investigate the nucleus at scales comparable to or less than the size of the SSPs to determine whether the SSPs are sampling typical or unusual areas. We estimate that a resolution of $<20 \mathrm{~cm}\left(10 \mathrm{~cm} \mathrm{px}^{-1}\right)$ is necessary to accomplish this, giving 5 resolution units across the SSPs.

The derivation of the total mass production rate from the comet is difficult to quantify from 2-D remote sensing of the dust and gas emission because a clear 3-D picture of the direction and velocity of outflowing material is not trivial to construct (Thomas et al., 1991). Furthermore, the spacecraft will only be operated for $\approx 8 \mathrm{~h} \mathrm{day}^{-1}$ and hence the flux can only be poorly determined for a limited time. The optimum way to determine the total mass loss from the comet is therefore to determine the change in the volume of the nucleus and use the bulk density derived from the radio science investigation (RSI) and the camera. An active area will lose around $2 \mathrm{~m}$ depth of material during the passage of the comet from aphelion to perihelion. To derive the total mass loss to an accuracy of $10 \%$ would then require a resolution of $\leq 20 \mathrm{~cm}$.

While the spacecraft can, in theory, go to within 1 nuclear radius of the surface, the operational strategy has not been decided at this stage of the mission design. The complexity can be illustrated by the following problem. The recent recovery of comet P/Wirtanen (Boehnhardt et al., 1996) at a visual magnitude of 21 has been interpreted as showing a very small, $\approx 700 \mathrm{~m}$ radius nucleus. This has prompted the suggestion that Rosetta could approach much closer than previously anticipated and hence, the resolution of the NAC could be reduced. This argument, however, ignores the consequences for the activity that Boehnhardt et al.'s observations imply. As Rickman and Jorda (this issue) point out, the observed water production rate of P/Wirtanen combined with the small nucleus implies that the surface has a very high active fraction. The nucleus was also observed to be active at around 3.2 AU - close to the heliocentric distance at which Rosetta will begin near-nucleus operations. Combined with the evidence for centimetre-sized particles in the vicinity of cometary nuclei (Campbell et al., 1989), this indicates that the spacecraft may be at risk (Kuehrt et al., 1996) and have to stay further away from the nucleus for a longer period than has been hitherto foreseen. This, in turn, suggests than even higher resolution may be required. 
The determination of the rotation periods of the nucleus (from $10^{4} \mathrm{~km}$ ) and of the asteroids (in order to establish the "ground truth" for ground-based asteroid pole determinations) and the requirement to detect $\mathrm{cm}$ sized particles in orbit about the nucleus also force the NAC to high resolution. We have concluded that a pixel scale of $20 \mu \mathrm{rad} \mathrm{px}^{-1}$ (a resolution of $20 \mathrm{~cm}_{\text {linepair }}^{-1}$ from $5 \mathrm{~km}$ ) is the most appropriate compromise between resolution and mass.

\section{Detector size and response.}

To produce high quality maps of the surface, and to provide accurate registration of images, a significant portion of images with adjacent footprints need to overlap, thereby replicating data. Large format $(2 \mathrm{k} x 2 \mathrm{k})$ CCDs have been selected for OSIRIS to minimize the waste caused by overlap and hence conserve probably the most precious resource on Rosetta, viz. telemetry. This leads to a field of view (FOV) of $2.35^{\circ}$ square at the required pixel scale. This value exceeds that needed to ensure that the nucleus will be in the FOV of the NAC at a distance of $10^{6} \mathrm{~km}$ (despite an error circle of $10^{4} \mathrm{~km}$ ) during the comet detection phase if OSIRIS is required for this purpose.

To reduce mass, devices with small pixels have been studied. A trade-off between development cost and mass indicates that $14 \times 14 \mu \mathrm{m}^{2}$ CCDs are appropriate. The high costs of CCD development and testing also suggest that the NAC and the WAC should have identical detectors. The scientific requirements for the investigation of gas emissions requires high UV quantum efficiency $(>30 \%)$ and high full-well to read noise ratio $\left(\approx 210^{4}\right)$. Consequently, backside illuminated devices with UV sensitive coatings have been baselined.

\section{The field of view of the WAC.}

The "Red Report" suggested a FOV for the WAC of around $17.2^{\circ}$. It was also suggested that a simpler design but with a similar FOV could accomplish navigation tasks when the spacecraft was close to the nucleus. However, in optimising our design we established that unless there was a strict requirement from the spacecraft that the WAC maintain the "Red Report" FOV for the purposes of serving as a back-up for the navigation camera, then good optical and straylight performance were of higher priority for the WAC than the FOV. We were particularly concerned that the wide FOV of the WAC in the "Red Report" would force upon us the use of much wider interference filters for gas emissions than desirable because of the convergence of the beam in the off-axis system. A $12^{\circ}$ square FOV has been selected. Use of identical detectors and readout electronics (appropriate for cost saving purposes) leads to a pixel scale for the WAC of $100 \mu \mathrm{rad} \mathrm{px}^{-1}$. With this system, the innermost coma within $10 \mathrm{~km}$ from the nucleus can be viewed from a cometocentric distance of only $100 \mathrm{~km}$ during the monitoring phase.

\section{Imaging speed.}

The high heliocentric distance and the speed of the motion of the spacecraft over the surface during the mapping phase drive the size of the optics of the system. Extensive calculations have shown that minimum exposure times of $\approx 50 \mathrm{~ms}$ with an F/8 telescope should cover most eventualities. A mechanical shutter will be employed which will give good image quality down to $10 \mathrm{~ms}$ (goal). The rate at which images need to be taken is driven by the requirement to have good phase angle coverage (in several colours) during the asteroid fly-bys and by the measurement of cometary outbursts by the WAC. Our current requirement is to image every $3.5 \mathrm{~s}$.

\section{Filter requirements and re-focussing.}

To provide a good selection of mineralogical and gas filters, places for 16 filters per camera have been reserved. The uncertainties in the reflectance properties of the target comet and the huge range in heliocentric distance that needs to be covered suggest that it is appropriate to include neutral density filters. A 
characteristic of the NAC optics is that if Rosetta were to make very close $(<2 \mathrm{~km})$ approachs to the nucleus, the NAC can go out of focus. Consequently, the optics will be focussed for a target at a distance of $4 \mathrm{~km}$ (not infinity as is usual with spacecraft-borne optical systems). Even with this adjustment, objects less than $2 \mathrm{~km}$ away from the spacecraft will still be defocussed. A place in the NAC filter wheel will therefore be reserved for a re-focussing lens which will give good images of the target even if the spacecraft approachs to a distance $1 \mathrm{~km}$ from the comet's surface.

The WAC gas band filters need to be as narrow as possible to minimize the contribution from the dust continuum. However, a lower limit of $\approx 4 \mathrm{~nm}$ is reached because of the convergence of the F/5.6 beam. Appropriate continuum filters will be carried for all investigated gas emission lines.

\section{$\underline{\mathbb{R} \text { requirements. }}$}

Several of the main constituents of cometary nuclei have characteristic broad $(\approx 0.1 \mu \mathrm{m})$ absorption bands in the near-infrared. Water ice, for example, has bands at 1.55 and $2.05 \mu \mathrm{m}$. The depths of these bands are highly sensitive to the mixing ratio of particulates (Clark et al., 1986). The distribution, homogeneity, and mixing ratio of these constituents in active and inactive regions is completely unknown. High temporal and spatial resolution can be used to investigate the changing properties of active regions throughout the escort phase and, particularly, during outbursts. The distribution of surface organics may be rather inhomogeneous and residues may be indicative of sites of previous emission. Imaging of the $3.4 \mu \mathrm{m}$ feature could therefore guide other experiments to regions of this type. In addition, 2-D imaging of major volatiles $\left(\mathrm{H}_{2} \mathrm{O}, \mathrm{CO}_{2}\right.$, and $\mathrm{CO}$ at $2.7,4.2$, and $4.7 \mu \mathrm{m}$, respectively) can provide major clues on the homogeneity of these species within active regions and on the gas flow-field within a few nuclear radii. It should also be noted that a rapid $\mathbb{R}$ imaging system may offer the optimum means of studying the surface composition of the asteroids during fast fly-bys.

These scientific objectives identify the need for high temporal (seconds), high spatial $\left(<1 \mathrm{~m} \mathrm{Ip}^{-1}\right)$, but low spectral resolution $(\lambda / \Delta \lambda=10)$ mapping of the surface of the target comet at infrared wavelengths between 1 and $4.8 \mu \mathrm{m}$.

\section{NAC DESIGN}

The optical layout of the NAC (without the IR system) is shown in Figure 1. It has been verified that the threemirror, anastigmatic, F/8 design (concave hyperbolic primary, convex parabolic secondary, and concave spherical tertiary) can be manufactured to the required tolerances and allows good baffling for straylight rejection. The focal plane assembly (FPA) can be placed either inside or outside the spacecraft hull depending upon the thermal properties of the spacecraft. Ideally, the CCD detectors need to be maintained at around 180 $\mathrm{K}$. At least three manufacturers have expressed interest in supplying the CCDs and have confirmed that our specifications can be met. Four parallel read-outs, a read-out noise of $<6 \mathrm{e}^{-}$at a rate of $300 \mathrm{kpx} \mathrm{s}^{-1}$ output $^{-1}$, and a full-well capacity of $120,000 \mathrm{e}^{-}$are required. The continuing convergence of the beam makes it appropriate to place the shutter and filter wheel as close to the detector as possible to minimize mass. A dual filter wheel concept (similar to that employed on the LASCO experiment on SOHO (Brueckner et al., 1995)) has been employed both to conserve volume (and mass) and to allow implementation of a neutral density filter and a re-focussing lens within the filter wheel. Eight positions in each wheel are foreseen. The selected filters are shown in Table 1. The shutter will be based on a design for the VIMS experiment on Cassini for which a prototype already exists. A front door cover, used to protect the optics from impacting dust particles and contamination has been included. This will be actuated by a stepper motor with a space-qualified, one shot wax device similar to that used by LASCO acting as a back-up. 


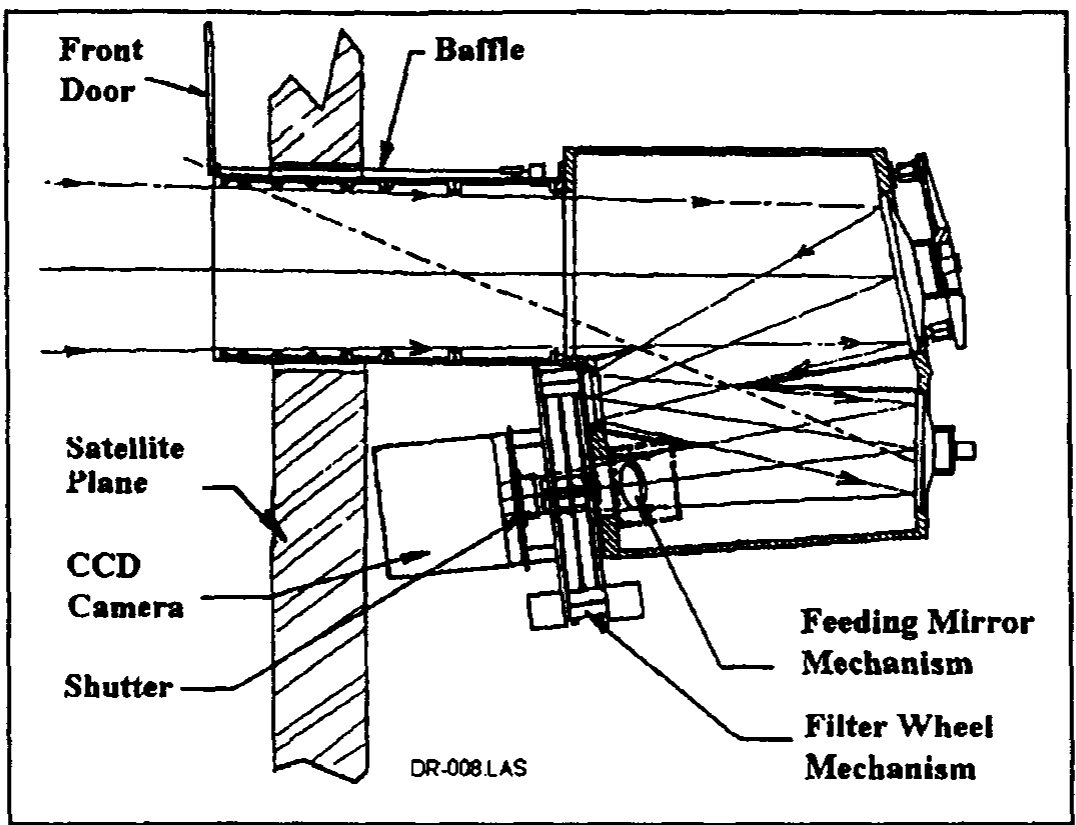

Figure 1 Optical layout of the NAC.

Table I NAC filters.

\begin{tabular}{|c|c|c|c|c|c|}
\hline Name & $\begin{array}{l}\text { Wavelength } \\
{[\mathrm{nm}]}\end{array}$ & $\begin{array}{c}\text { Bandwidth } \\
{[\mathrm{nm}]}\end{array}$ & $\begin{array}{c}\text { Peak } \\
\text { Trans } \\
{[\%]}\end{array}$ & Objective & Wheel \\
\hline Plate & $600( \pm 25)$ & $>600$ & $>90$ & $\begin{array}{l}\text { Empty position or transparent material to allow } \\
\text { use of wheel } 2\end{array}$ & 1 \\
\hline Plate & $600( \pm 25)$ & $>600$ & $>90$ & $\begin{array}{l}\text { Empty position or transparent material to allow } \\
\text { use of wheel } 1\end{array}$ & 2 \\
\hline Neutral & - & - & 2 & $2 \%$ transmission neutral density filter & 1 \\
\hline Re-focus & $600( \pm 25)$ & $>600$ & $>90$ & Refocusing lens for near-nucleus imaging & 1 \\
\hline Far-UV & $270( \pm 5)$ & $50( \pm 5)$ & $>50$ & Surface spectral reflectance & 2 \\
\hline $\mathrm{OH}-\mathrm{NAC}$ & $308( \pm 0.5)$ & $5( \pm 0.5)$ & $>25$ & $\begin{array}{l}\text { Detection of } \mathrm{OH} \text { emission from the vicinity of the } \\
\text { nucleus }\end{array}$ & 1 \\
\hline $\mathrm{OH}$-cont & $325( \pm 1)$ & $10( \pm 1)$ & $>40$ & $\begin{array}{l}\text { Continuum filter for dust subtraction from } \mathrm{OH} \\
\text { filter image }\end{array}$ & 1 \\
\hline Near-UV & $359( \pm 5)$ & $60( \pm 5)$ & $>50$ & Surface spectral reflectance; ECAS u filter & 2 \\
\hline Blue & $480( \pm 5)$ & $80( \pm 5)$ & $>70$ & Surface spectral reflectance & 2 \\
\hline Green & $550( \pm 5)$ & $57( \pm 5)$ & $>70$ & Surface spectral reflectance; ECAS v filter & 2 \\
\hline Orange & $645( \pm 5)$ & $94( \pm 5)$ & $>80$ & HMC orange filter; surface spectral reflectance & 2 \\
\hline Hydra & $700( \pm 2)$ & $20( \pm 3)$ & $>50$ & Water of hydration band & 1 \\
\hline Red & $740( \pm 5)$ & $60( \pm 5)$ & $>70$ & Surface spectral reflectance & 2 \\
\hline Ortho & $780( \pm 2)$ & $20( \pm 3)$ & $>50$ & Orthopyroxene & 1 \\
\hline Near-IR & $840( \pm 5)$ & $90( \pm 5)$ & $>70$ & Surface spectral reflectance & 2 \\
\hline Olivine & $890( \pm 2)$ & $20( \pm 3)$ & $>50$ & Olivine & 1 \\
\hline
\end{tabular}

\section{WAC DESIGN}

The WAC (Figure 2) is a two-mirror (oblate ellipsoid, convex primary, oblate ellipsoid, concave secondary), off-axis, F/5.6 system (Ragazzoni et al., 1995; Naletto et al., 1995). The filter wheel, shutter, front cover, and 
FPA are all similar to those included in the NAC. The filters to be incorporated in the WAC filter wheel are shown in Table 2. The positioning of the WAC optics and FPA can be essentially independent of the positioning of the NAC. Furthermore, the This has some significance since the proposed IR option requires the NAC optics to be at a lower temperature $(240 \mathrm{~K})$ than the WAC optics and $s \approx 290 \mathrm{~K})$ and thus the both from the spacecraft and from each other. Furthermore, the detectors need to be isolated thermally from the optics.

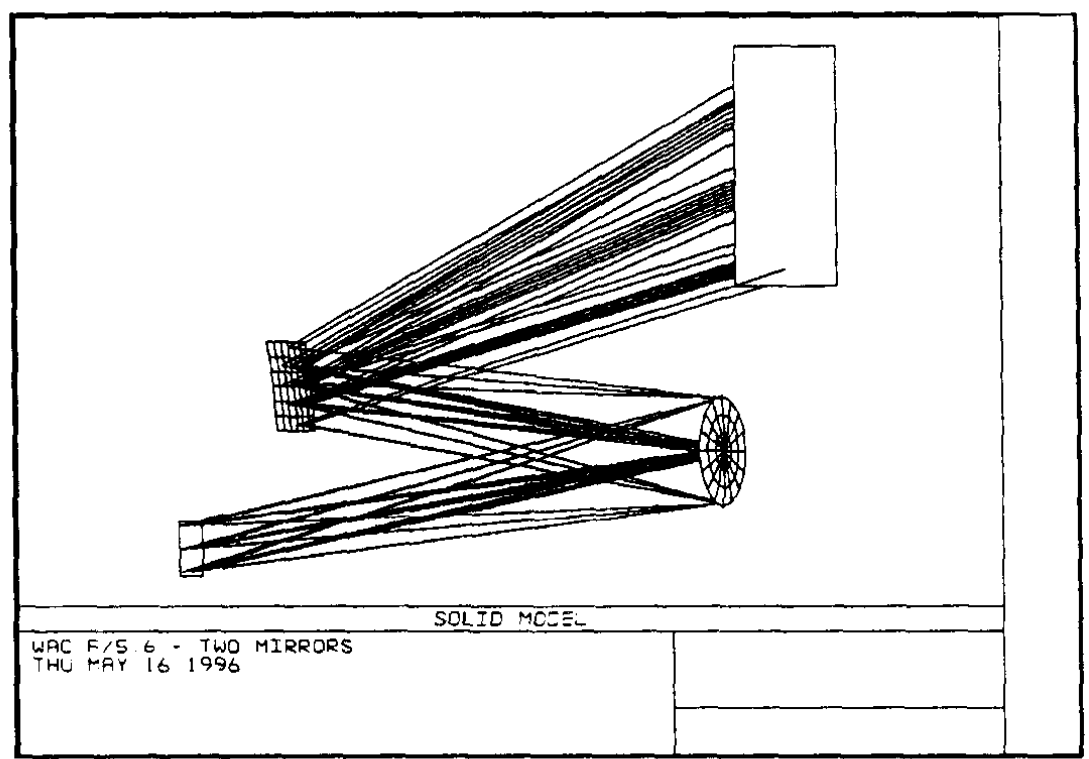

Figure 2 Ray trace diagram of the WAC optics

Table II WAC filters.

\begin{tabular}{|c|c|c|c|c|c|}
\hline Name & $\begin{array}{c}\text { Wavelength } \\
\text { [nm] }\end{array}$ & $\begin{array}{c}\text { Bandwidth } \\
\text { [nm] }\end{array}$ & $\begin{array}{c}\text { Peak } \\
\text { Trans. } \\
{[\%]}\end{array}$ & Objective & Wheel \\
\hline Plate & $600( \pm 50)$ & $>600$ & $>90$ & $\begin{array}{l}\text { Empty position or transparent material to allow use of filter } \\
\text { wheel } 2\end{array}$ & 1 \\
\hline Plate & $600( \pm 50)$ & $>600$ & $>90$ & $\begin{array}{l}\text { Empty position or transparent material to allow use of filter } \\
\text { wheel } 1\end{array}$ & 2 \\
\hline Neutral & - & - & 2 & $2 \%$ transmission neutral density filter & 1 \\
\hline UV245 & $245( \pm 2)$ & $15( \pm 2)$ & $>35$ & Continuum; backup surface spectral reflectance & 1 \\
\hline CS & $257( \pm 0.5)$ & $4( \pm 0.5)$ & $>25$ & CS gas emission & 1 \\
\hline UV295 & $295( \pm 2)$ & $10( \pm 2)$ & $>35$ & Continuum for $\mathrm{OH}$ & 1 \\
\hline $\mathrm{OH}-\mathrm{WAC}$ & $308( \pm 0.5)$ & $4( \pm 0.5)$ & $>25$ & Detection of $\mathrm{OH}$ emission from the vicinity of the nucleus & 1 \\
\hline UV325 & $325( \pm 2)$ & $10( \pm 2)$ & $>35$ & Continuum for $\mathrm{OH}$; backup surface spectral reflectance & 1 \\
\hline $\mathrm{NH}$ & $336( \pm 0.5)$ & $4( \pm 0.5)$ & $>25$ & $\mathrm{NH}$ gas emission & 1 \\
\hline UV375 & $375( \pm 2)$ & $10( \pm 2)$ & $>50$ & Continuum for $\mathrm{CN}$; backup surface spectral reflectance & 2 \\
\hline $\mathrm{CN}$ & $388( \pm 0.5)$ & $4( \pm 0.5)$ & $>25$ & $\mathrm{CN}$ gas emission & 2 \\
\hline OI & $630( \pm 0.5)$ & $4( \pm 0.5)$ & $>25$ & $\mathrm{O}\left({ }^{\prime} \mathrm{D}\right)$ gas emission for dissociation of $\mathrm{H}_{2} \mathrm{O}$ & 2 \\
\hline VIS655 & $655( \pm 2)$ & $10( \pm 2)$ & $>50$ & Continuum for $\mathrm{NH}_{2}$; backup surface spectral reflectance & 2 \\
\hline $\mathrm{NH}_{2}$ & $670( \pm 0.5)$ & $4( \pm 0.5)$ & $>25$ & $\mathrm{NH}_{2}$ gas emission & 2 \\
\hline Johnson $\mathrm{R}$ & $750( \pm 30)$ & $200( \pm 30)$ & $>90$ & $\begin{array}{l}\text { Broad-band } \mathrm{R} \text { filter for nucleus and asteroid detection in case } \\
\text { of NAC failure }\end{array}$ & 2 \\
\hline IR855 & $855( \pm 2)$ & $10( \pm 2)$ & $>50$ & Backup surface spectral reflectance & 2 \\
\hline
\end{tabular}


The analogue read-out electronics for the CCDs will pass the acquired data to 14-bit analogue to digital converters (ADCs). The output will then be passed to the data processing unit (DPU). The DPU not only needs to control the experiment but must also match a data acquisition rate of around $32 \mathrm{Mbit} \mathrm{s}^{-1}$ with a telemetry rate of $\approx 8 \mathrm{kbit} \mathrm{s}^{-1}$. A dual redundant system with a $3.0 \mathrm{Gbit}$ mass memory is foreseen. A meta command language similar to that developed for the SUMER experiment on SOHO will be used. Sophisticated data selection and compression techniques (including discrete wavelet transform encoding) are envisaged. The mechanisms will be driven by a microcontroller board which will also provide instrument housekeeping data acquisition. A dual redundant power supply will be used. A detailed radiation design will be performed to assess requirements for spot shielding of radiation sensitive parts.

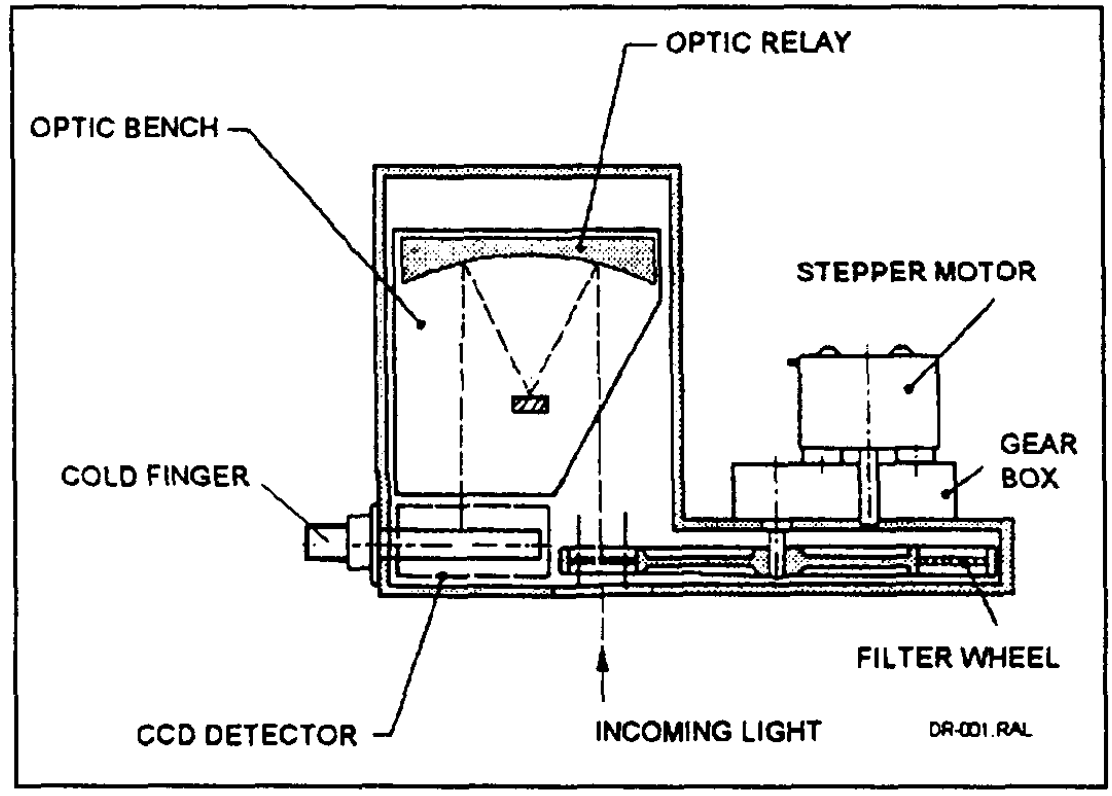

Figure 3 Schematic diagram of the IR channel.

\section{IR OPTION}

The optics of the IR imaging system (Figure 3) will be fed by a flip feed mirror within the NAC. The incoming light will pass through a filter wheel thereby entering a cold trap which is maintained at $130 \mathrm{~K}$ by passive cooling to a $0.5 \mathrm{~m}^{2}$ radiator. The filter wheel will probably contain fixed inter-ference filters giving a $\lambda / \Delta \lambda$ of 10. Circular variable filters (CVFs) have been considered but their throughput may be inadequate at this stage of their development. The filter wheel system will be similar to that for the NAC and the WAC but modified for cold temperatures. Specific filters to investigate $\mathrm{H}_{2} \mathrm{O}(2.7 \mu \mathrm{m})$ and $\mathrm{CO}_{2}(4.2 \mu \mathrm{m})$ gas emission and the associated continuum will be carried. The re-imaging optics provides no change in the image scale but is used solely to minimize the thermal background reaching the detector. A mercury cadmium telluride (MCT) detector $\left(640 \times 480 \mathrm{px}^{2}, 27 \times 27 \mu \mathrm{m}^{2}\right)$ with high full-well capacity will be used giving an image scale of $38.6 \mu \mathrm{rad} \mathrm{px}^{-1}$. Rapid read-out through four $12 \mathrm{bit}$ ADCs is foreseen giving $1000 \mathrm{e}^{-}$read noise at $400 \mathrm{kpx} \mathrm{s}^{-1}$ output $^{-1}$. The exact wavelength range to be covered is (at the time of writing) subject to a tradeoff study but will extend from $1 \mu \mathrm{m}$ to at least $3.5 \mu \mathrm{m}$ and possibly $4.8 \mu \mathrm{m}$ (goal).

\section{POWER AND MASS BUDGETS}

The current mass budget for the entire OSIRIS system is $23.1 \mathrm{~kg}$ (not including a $15 \%$ margin). The power requirement is $32.4 \mathrm{~W}$ although a low power mode (drawing $10.4 \mathrm{~W}$ by using only $1 \mathrm{CCD}$ and 1 output) is foreseen which could allow early switch-on of the experiment at large heliocentric distances. 


\section{IMPLEMENTATION}

The OSIRIS experiment will be built by a consortium comprising seven countries. Each country is represented by a Lead Scientist (LS) who forms the single point interface to the Principal Investigator (PI). Within each country, several organisations will contribute to the experiment. The countries and organisations are given in Table 3.The present breakdown of responsibilities is given in Table 4. At the time of writing, the interface definitions are proceeding smoothly and work has begun on detailed designs of the separate components.

Table III The organisations contributing to OSIRIS

\begin{tabular}{||l|l|}
\hline Country & Contributing Organisations \\
\hline Germany & $\begin{array}{l}\text { Max-Planck-Institut für Aeronomie, Katlenburg-Lindau } \\
\text { (MPAE) } \\
\text { Technical University, Braunschweig (IDA) } \\
\text { DLR-Institut für Weltraumsensorik, Berlin (DLR-WS) }\end{array}$ \\
\hline France & \begin{tabular}{l} 
Laboratoire d'Astronomie Spatiale, Marseille (LAS) \\
\hline Italy
\end{tabular} \\
\hline Spain & $\begin{array}{l}\text { University of Padova (UPD) } \\
\text { National Institute for Aerospace Technology (INTA) } \\
\text { Universidad Politecnica Madrid (UPM) }\end{array}$ \\
\hline Belgium & Belgian Institute for Space Aeronomy, Brussels (BIRA) \\
\hline Sweden & Astronomical Observatory, Uppsala (AOU) \\
\hline ESA & Space Science Dept. of ESA, ESTEC (SSD) \\
\hline
\end{tabular}

Table IV OSIRIS task distribution

\begin{tabular}{||l|l|}
\hline Task & $\begin{array}{l}\text { Responsible } \\
\text { Institute }\end{array}$ \\
\hline $\begin{array}{l}\text { Overall responsibility, CCDs, electronics, } \\
\text { DPU, integration, calibration, operation }\end{array}$ & MPAE \\
\hline Narrow angle camera & LAS \\
\hline IR channel & BIRA \\
\hline Wide angle camera & UPD \\
\hline Thermal design & UPM \\
\hline Data processing unit & SSD \\
\hline Filter wheels, and power supply & INTA \\
\hline Mechanism controller & IAA \\
\hline Shutters & UPD \\
\hline Filters & AOU \\
\hline
\end{tabular}




\section{CONCLUSIONS}

The OSIRIS experiment is a scientific imaging system, specifically designed to investigate the nucleus and environment of a short-period comet. The present design promises to provide a significant leap forward in the investigation of the most primitive objects in our Solar System and is an instrument worthy of taking its place on an ESA cornerstone mission. 